Jariot Garcia, M., Laborda Molla, C. y González Fernández, H. (2020). El perfil competencial laboral de personas con discapacidad intelectual en centros ocupacionales. Revista de Investigación Educativa, 38(2), 475-493.

DOI: http://dx.doi.org/10.6018/rie.312241

\title{
El perfil competencial laboral de personas con discapacidad intelectual en centros ocupacionales
}

\section{The labour competence profile of people with intellectual disabilities in occupational centres}

\author{
Mercè Jariot Garcia, Cristina Laborda Molla y Hernar González Fernández \\ Departamento de Pedagogía Aplicada, Facultad de Ciencias de la Educación, \\ Universidad Autónoma de Barcelona (España)
}

\begin{abstract}
Resumen
La inserción laboral repercute positivamente en el entorno del colectivo de personas con discapacidad intelectual (DI), y el empleo es decisivo en su proceso de integración social y participación comunitaria. Se pretenden optimizar los procesos de integración laboral y desarrollo profesional de las personas con DI leve y moderada para facilitar su inclusión social. Se realizó un muestreo no probabilístico de tipo incidental, en el que participaron 16 centros ocupacionales de la provincia de Barcelona. Se creó y aplicó un cuestionario a 100 personas con grados de DI leve y moderado. Se utilizó un diseño de investigación no experimental, en el que se analizaron las competencias profesionales de los participantes en su contexto natural (centros ocupacionales). Se constata una relación (positiva y moderada) estadísticamente significativa entre todas las competencias. Las personas con DI tienen un desarrollo similar en las competencias analizadas; las que presentan un grado de DI leve muestran niveles competenciales superiores a los de las personas con DI moderada. Es destacable que, independientemente del grado, todas las competencias evaluadas superen la puntuación media. Seguramente el hecho de que las personas analizadas estén ubicadas en centros ocupacionales es una variable que facilita su desarrollo competencial.

Palabras clave: discapacidad intelectual; inclusión; desarrollo profesional; formación profesional.
\end{abstract}

Correspondencia: Mercè Jariot Garcia, merce.jariot@uab.cat, Pl. del Coneixement, Campus de la UAB, 08193 Bellaterra (Cerdanyola del Vallès) 


\begin{abstract}
Labour integration has a positive impact on people with Intellectual Disability (ID), being employment a key element in their process of social integration and community participation. The aim of this research is to optimize the labour integration and professional development of people with mild and moderate ID in order to facilitate their social inclusion. A non-probabilistic incidental sampling was carried out, with the participation of 16 occupational centres of Barcelona's metropolitan area. A questionnaire was created and administered to 100 people with mild and moderate ID. A non-experimental research design was used, in which the professional skills of the participants in their natural professional context (occupational centres) were analysed. Results show a statistically significant relationship (positive and moderate) between all the competences. People with ID have a similar development in the analysed competences; those with a low degree of ID show higher levels of competence than those with moderate ID. It is noteworthy that, regardless of the degree, all the evaluated competences exceed the average score. The fact that the participants in the research are located in occupational centres is certainly a variable that facilitates their competence development.

Keywords: intellectual disability; inclusion; professional development; professional training.
\end{abstract}

\title{
Introducción
}

El trabajo es uno de los indicadores más importantes de la calidad de vida de las personas (Simões y Santos 2016), puesto que incrementa las relaciones sociales, proporciona independencia y autonomía económica y mejora la confianza y la seguridad en uno mismo (Pegalajar y Xandri, 2015).

El trabajo es igualmente un derecho de todas las personas al que, en la situación actual de políticas de desregulación y flexibilidad laboral, es complicado acceder y mantener. Dichas políticas han propiciado altas tasas de empleo temporal, reducción de salarios y demandas de cualificación profesional (Arancibia, 2011) así como cambios constantes en los índices de paro generales (18,91\% en el tercer trimestre de 2016) (Instituto Nacional de Empleo, 2016). Por cierto, que las estadísticas se complican aún más al hablar de personas con discapacidad, cuya tasa de paro en el año 2015 fue más de 10 puntos superior al de los trabajadores sin discapacidad (Observatorio sobre Discapacidad y Mercado de Trabajo en España, 2016).

El entorno laboral actual está igualmente caracterizado por la segmentación y polarización del trabajo, donde tal y como describen De la Fuente y González-Castro (2009), conviven un mercado primario que ofrece empleos estables y condiciones aceptables de trabajo y promoción, y otro secundario con puestos de trabajo precarios e inestables y malas condiciones laborales, donde la tendencia es el cambio en las condiciones de contratación reguladas por las necesidades de las empresas (De Cuyper, Mauno, Kinnunen y Mäkikangas, 2011).

En este contexto se exigen nuevos conocimientos y competencias para la consecución y el mantenimiento de un empleo y para la promoción laboral (Martínez-Agut y Ramos Hernando, 2014), que permitan a los trabajadores adaptarse a los cambios y adquirir nuevos conocimientos de forma constante (Comisión Europea, 2008). Aquí 
es donde entran en juego las competencias que necesitan las personas para adaptarse al entorno y poder obtener y conservar un trabajo (Martínez-Clares, Martínez-Juárez y Muñoz-Cantero, 2008), y el dominio de otras competencias laborales para actuar con eficacia en situaciones profesionales de desarrollo personal (Alonso-Marín, 2010).

Especificando en el colectivo de personas con discapacidad de tipo intelectual, la importancia de la inserción laboral repercute positivamente en el entorno en el que se desenvuelve (Pegalajar y Xandri, 2015), siendo el empleo un elemento decisivo en el proceso de integración social y participación en la comunidad (Ali, Schur y Blanck, 2011). En esta línea, Flores, Jenaro, Garcia-Calvo y González-Gil (2017) introducen la calidad de vida laboral como nuevo elemento de la calidad de vida de la persona, aunque tradicionalmente ambos conceptos se hayan utilizado como similares.

Autores como Roselló y Verger (2008) afirman que existe una relación directa entre las capacidades de cada persona y el grado de discapacidad intelectual (DI) y las posibilidades de obtener un empleo, por lo que es necesario conocer cuáles son las competencias laborales que poseen dichas personas para facilitar su inserción laboral. Por otro lado, González y Laborda (2017) establecen una relación significativa entre las competencias laborales, el nivel de DI y la calidad de vida en dicho colectivo.

El actual mercado laboral demanda historiales profesionales brillantes, experiencia laboral y competencias profesionales a todos los trabajadores; no obstante, si el candidato es una persona con una DI, el proceso se complica puesto que existe un conjunto de dificultades específicas para formarse laboralmente, y encontrar y mantener un puesto de trabajo (Pallisera, Vila y Valls, 2003). De hecho, las personas con DI requieren, en la mayoría de las ocasiones, de adaptaciones y cambios en su entorno laboral y sus puestos de trabajo adquieren características diferenciales (Laborda y González, 2017).

Autores como Castro, Casas, Sánchez, Vallejos, y Zúñiga (2016), consideran que este colectivo necesita de modelos que promuevan la adquisición de competencias clave que favorezcan su inserción laboral desde una visión inclusiva de la sociedad y de la persona, y que promuevan la vida independiente.

\section{Método}

\section{Objetivos}

El objetivo general del estudio es el de optimizar los procesos de integración laboral y desarrollo profesional de las personas con DI para facilitar su inclusión social.

Para ellos, se concretaron los siguientes objetivos específicos:

Conocer el nivel de desarrollo de competencias laborales de los usuarios de centros ocupacionales.

- Observar si existen diferencias en el nivel de desarrollo de dichas competencias en función de las variables género y grado de discapacidad intelectual.

- Extraer claves educativas para la facilitación de la integración y desarrollo laboral de las personas con DI. 


\section{Población y Muestra}

Ante la imposibilidad de realizar un muestreo aleatorio efectivo que garantizara la representatividad de la muestra, se optó por realizar un muestreo no probabilístico de tipo incidental. Se solicitó para ello la participación voluntaria de los profesionales de 30 centros ocupacionales a través de un cuestionario presencial. De los contactados inicialmente, 16 accedieron a participar en la investigación. El criterio de elección correspondió a la distribución de centros públicos/privados de la zona y a la posterior voluntariedad de los mismos. Así, el 7\% de los centros eran de carácter público, y el 93\% privado, distribución similar a la poblacional en la que el 10\% son públicos (19) y el $90 \%$ privados (166).

Las condiciones de las personas con DI para participar en la investigación eran llevar más de 1 año realizando la misma tarea laboral, tener un buen nivel comunicativo y tener un nivel de discapacidad intelectual leve o moderada.

Cada centro seleccionó 5 personas en función de los criterios expuestos. Los evaluadores internos debían tener una antigüedad en el centro de 1 año o superior, y llevar 6 meses como mínimo trabajando con los usuarios que debían evaluar. Los participantes fueron un total de 100 usuarios, de edades comprendidas entre los intervalos de 20-29 un $24 \%$; 30-39 un 36\%; $40-49$ un $14 \%$, 50-59 un $16 \%$ y más de 60 un $10 \%$.

Del total de la muestra 48 fueron hombres y 52 mujeres, 39 con un grado de DI leve y 61 moderado. El 17\% de la muestra la conforman hombres con un grado de DI leve, el 31\% hombres con un grado de discapacidad moderado, el 22\% mujeres con un grado de DI leve y el 30\% mujeres con un grado de DI moderado.

\section{Instrumento}

Para el desarrollo de la investigación se creó un cuestionario adhoc que evaluaba el desarrollo de las competencias laborales, clasificadas en cuatro tipos: cognitivas y de aprendizaje, funcionales, sociales y actitudinales y comunicativas, basadas en los trabajos de Lucas, Arias y Ovejero (2005) y Docampo y Morán (2014). El primero explora los factores relacionados con la obtención y el mantenimiento de empleo de las personas con discapacidad, y los encuadra en los siguientes: competencias cognitivas y hábitos de trabajo, habilidades sociales, interés de la persona, capacidad de adaptación y de aceptación de la autoridad. El segundo sugiere explorar las competencias laborales a través de sus dos saberes: el saber hacer y el saber estar.

El cuestionario resultante se dividió en dos partes: en la primera se recogía información referente a variables descriptivas; sexo, edad, grado de discapacidad intelectual y centro de trabajo. En la segunda, se concentraban los ítems referentes a todas las variables que fueron determinadas a partir de cuatro bloques competenciales, constituidos por los factores que conformaron esta investigación y que se presentan en la tabla 1. Todos los ítems debían ser fácilmente observables por el responsable del trabajador, que debía evaluarlos en una escala del 1 al 4, siendo 1 el desarrollo más bajo y 4 el superior.

Previo a la aplicación del instrumento, se realizó un estudio de las características psicométricas del mismo y se recogieron evidencias sobre la fiabilidad y la validez de la medida que proporcionaba. 
El proceso de validación se realizó en base a una administración (lectura en profundidad) a un grupo de expertos, constituido por 6 profesionales que trabajan en centros ocupacionales, que ayudaron a perfilar el lenguaje empleado en el cuestionario, manteniéndose las competencias inicialmente propuestas.

Asimismo, se comprobó que presentaba una consistencia interna aceptable (a de Cronbach de .913) y en, cada una de las subescalas, oscilaron entre .718 y .855, tal y como puede observarse en la tabla 2.

Tabla 1

Bloques y competencias incluidas en el cuestionario

\begin{tabular}{|c|c|c|c|c|c|}
\hline \multirow{5}{*}{ 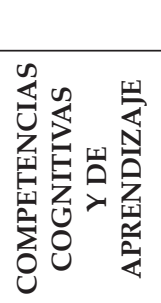 } & \multirow[b]{2}{*}{ Atención selectiva } & \multicolumn{4}{|c|}{ ESCALA } \\
\hline & & 1 & 2 & 3 & 4 \\
\hline & Atención sostenida & 1 & 2 & 3 & 4 \\
\hline & Detección de errores & 1 & 2 & 3 & 4 \\
\hline & Potencial de aprendizaje & 1 & 2 & 3 & 4 \\
\hline \multirow{6}{*}{ 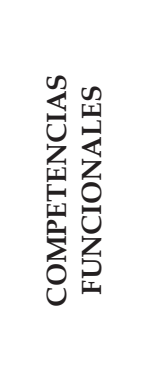 } & Capacidad para sistematizar las tareas & 1 & 2 & 3 & 4 \\
\hline & Planificación y organización & 1 & 2 & 3 & 4 \\
\hline & Resistencia delante de las tareas repetitivas & 1 & 2 & 3 & 4 \\
\hline & Resolución de problemas & 1 & 2 & 3 & 4 \\
\hline & Capacidad para solicitar ayuda cuando lo necesite & 1 & 2 & 3 & 4 \\
\hline & Habilidades manipulativas & 1 & 2 & 3 & 4 \\
\hline \multirow{11}{*}{ 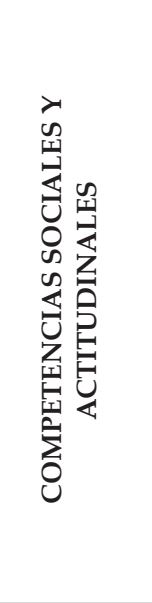 } & Relación con los profesionales & 1 & 2 & 3 & 4 \\
\hline & Relación con los compañeros & 1 & 2 & 3 & 4 \\
\hline & Capacidad de trabajo en equipo & 1 & 2 & 3 & 4 \\
\hline & Respetar y cuidar los materiales / herramientas & 1 & 2 & 3 & 4 \\
\hline & Nivel de responsabilidad & 1 & 2 & 3 & 4 \\
\hline & Nivel de iniciativa & 1 & 2 & 3 & 4 \\
\hline & Aceptación de les críticas & 1 & 2 & 3 & 4 \\
\hline & Nivel de autonomía & 1 & 2 & 3 & 4 \\
\hline & Aceptación de la autoridad & 1 & 2 & 3 & 4 \\
\hline & Nivel de flexibilidad y de adaptación a los cambios & 1 & 2 & 3 & 4 \\
\hline & Capacidad de motivación & 1 & 2 & 3 & 4 \\
\hline
\end{tabular}




\begin{tabular}{|c|c||c|c|c|c|}
\cline { 2 - 6 } \multicolumn{1}{c|}{} & \multicolumn{3}{|c|}{ ESCALA } \\
\hline \multirow{2}{*}{} & Comprensión de instrucciones verbales & 1 & 2 & 3 & 4 \\
\cline { 2 - 6 } & Comprensión de las instrucciones escritas & 1 & 2 & 3 & 4 \\
\cline { 2 - 7 } & Expresión oral & 1 & 2 & 3 & 4 \\
\cline { 2 - 6 } & Expresión escrita & 1 & 2 & 3 & 4 \\
\hline
\end{tabular}

Tabla 2

Resultados a de Cronbach general y por subescalas

\begin{tabular}{lcc}
\hline \multicolumn{1}{c}{ Dimensiones } & Número de ítems & Alfa de Cronbach \\
\hline Escala total & 25 & .913 \\
Cognitivas y de aprendizaje & 4 & .795 \\
Funcionales & 6 & .718 \\
Sociales y actitudinales & 11 & .855 \\
Comunicativas & 4 & .729 \\
\hline
\end{tabular}

\section{Procedimiento de recogida y análisis de datos}

La comunicación con los centros ocupacionales se realizó vía telefónica y mediante correo electrónico. En ambos casos se explicaba brevemente el objetivo de la investigación.

Con los 16 que accedieron a participar se concretó un encuentro presencial en cada centro con un miembro del equipo directivo. En dicha reunión se explicó con detalle la investigación, y se organizó el proceso de administración y el de recogida de datos. Así mismo, se dejaron los instrumentos para que el centro pudiera aplicarlos cuando más le conviniera.

Pasado el periodo pactado de 1 mes, uno de los investigadores pasó por los centros para recoger los cuestionarios, avisando con anterioridad telefónicamente.

Se utilizó un diseño de investigación no experimental, en el que se analizaron las competencias profesionales de los participantes en su contexto natural (centros ocupacionales).

Inicialmente se llevó a cabo un análisis de tipo descriptivo. Se calcularon las medias, desviaciones típicas y medianas de todos los ítems del cuestionario. Así mismo se realizaron representaciones de distribuciones de frecuencias. Posteriormente se analizaron las relaciones existentes entre los cuatro niveles competenciales ( $\mathrm{r}$ de Pearson). También se desarrolló un análisis de los datos mediante la prueba t-test para grupos 
independientes, para comprobar las diferencias en el desarrollo de las competencias entre las personas con DI leve y moderado.

Por último, y para comprobar si existían o no diferencias en cuanto al nivel competencial entre hombres y mujeres con DI leve y moderado, se planteó un Análisis de Varianza (ANOVA) de un factor con cuatro niveles, en el que el factor estaba compuesto por la variable cualitativa género y DI, con cuatro niveles (hombres con DI leve, hombres con DI moderada; mujeres con DI leve y mujeres con DI moderada), las variables respuesta cuantitativas eran: el nivel general de competencias laborales y el nivel competencial en cada una de las subescalas del cuestionario. Se realizó también el contraste de Levene sobre la igualdad de las varianzas de error, para contrastar la hipótesis de que la varianza de error de la variable dependiente era igual a lo largo de todos los grupos. Tras la realización de los contrastes se procedió a la realización de comparaciones múltiples (pruebas post hoc) mediante la DHS de Tukey para determinar en qué escalas concretas se producían diferencias entre hombres con DI leve, hombres con DI moderado, mujeres con DI leve y mujeres con DI moderado.

Se empleó el paquete estadístico SPSS versión 21 para Windows para el análisis de los datos.

El diseño de investigación contempló los permisos y las recomendaciones éticas, puesto que el tratamiento de los datos fue siempre anónimo y se contó con la aprobación de los centros implicados.

\section{Resultados}

\section{Resultados competenciales globales}

Los resultados generales demuestran que el desarrollo de las 4 competencias analizadas en los sujetos de la muestra es similar. Las puntuaciones medias se sitúan entre el 2,60 (para las funcionales y comunicativas) y 2,66 (para las cognitivas y de aprendizaje y sociales y actitudinales).

Analizando estos datos en función del grado de DI (figura 1), se aprecian algunas diferencias:

- Las personas con DI leve tienen un desarrollo superior que las de grado moderado en todas las competencias analizadas.

- Las personas con DI moderada muestran un desarrollo similar en todas ellas. 


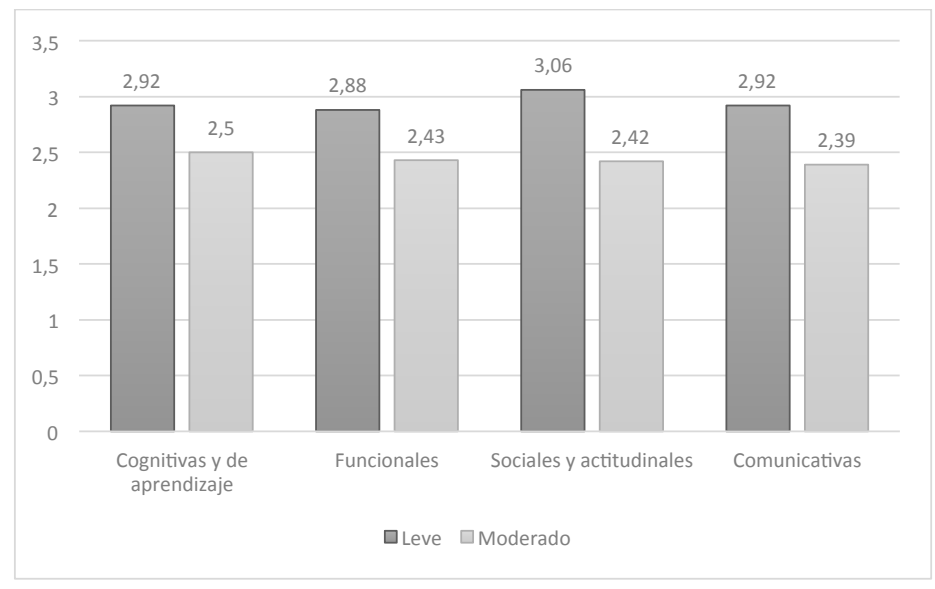

Figura 1. Niveles de competencia según el grado de DI.

A continuación, se entra en el análisis específico de los resultados de los 4 bloques de competencias.

\section{Resultados según el nivel de competencias cognitivas y de aprendizaje}

En la figura 2 se observa que, si tenemos en cuenta que la puntuación máxima de la escala es de 4, todas las puntuaciones relacionadas con las competencias cognitivas y de aprendizaje de ambos grupos están por encima de la media. La atención selectiva es la competencia más alta entre todos los participantes mientras que las menos desarrolladas son la detección de errores para las personas con DI leve y el potencial de aprendizaje para las de DI moderado. En todas las competencias las personas con un grado de DI leve muestran un nivel ligeramente superior.

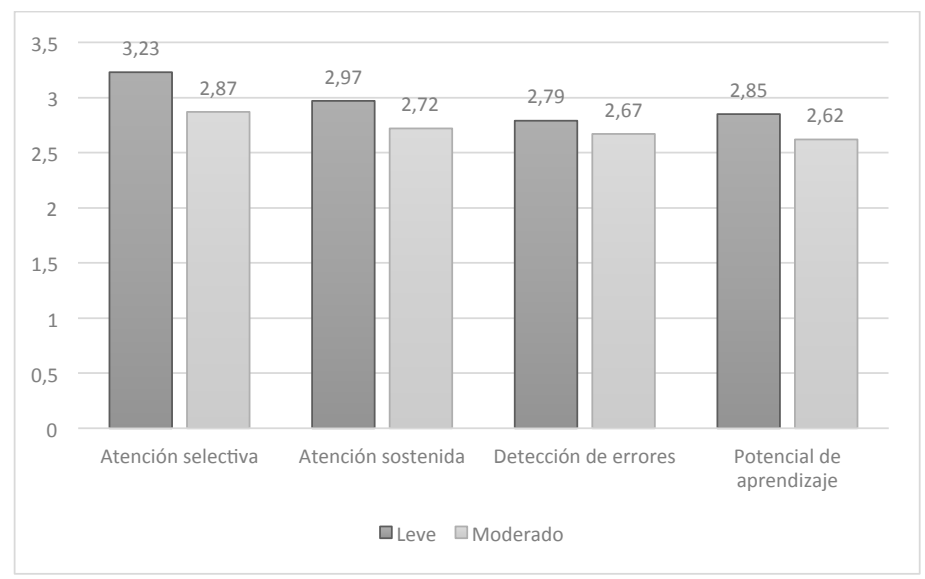

Figura 2. Competencias cognitivas y de aprendizaje. 


\section{Resultados según el nivel de competencias funcionales}

En la figura 3 se observa que todas las puntuaciones relacionadas con las competencias funcionales están por encima de la media, que las competencias más altas para el grupo de usuarios con DI leve es la capacidad de solicitar ayuda cuando se necesita, mientras que en el caso de los de DI moderado son las relacionadas con habilidades manipulativas. Las más bajas son la resistencia frente tareas repetitivas y la capacidad de solucionar problemas respectivamente. Tal y como puede observarse las personas con un grado de discapacidad leve muestran en todos los casos un nivel ligeramente superior.

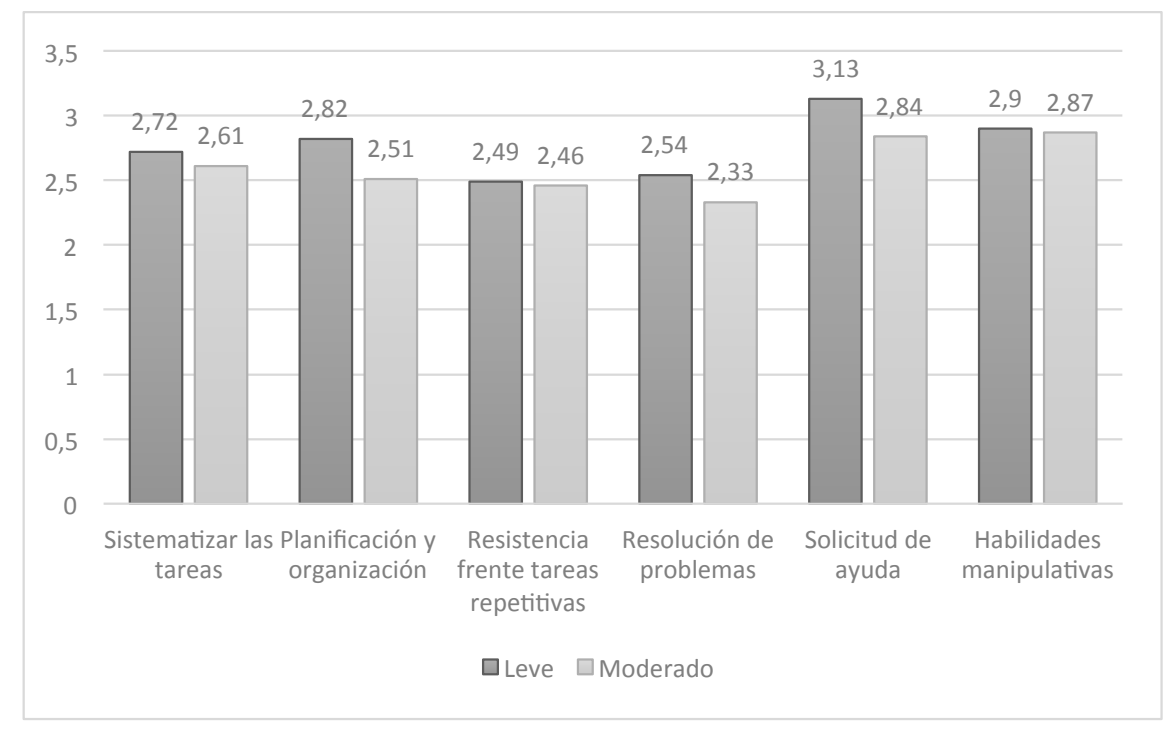

Figura 3. Competencias funcionales.

\section{Resultados según el nivel de competencias sociales y actitudinales}

En la figura 4 se observa que todas las puntuaciones relacionadas con las competencias sociales y actitudinales están por encima de la media. Las personas con un grado de discapacidad leve tienen un nivel ligeramente superior en todas las competencias excepto en la relación con los compañeros, donde los superan las personas con un grado de discapacidad moderado. En ambos grupos coinciden las puntuaciones más altas (relación con los profesionales), y las más bajas (aceptación de la crítica).

Por último, la figura 4 refleja que las personas con grado de discapacidad leve muestran un nivel bastante superior en la iniciativa. 


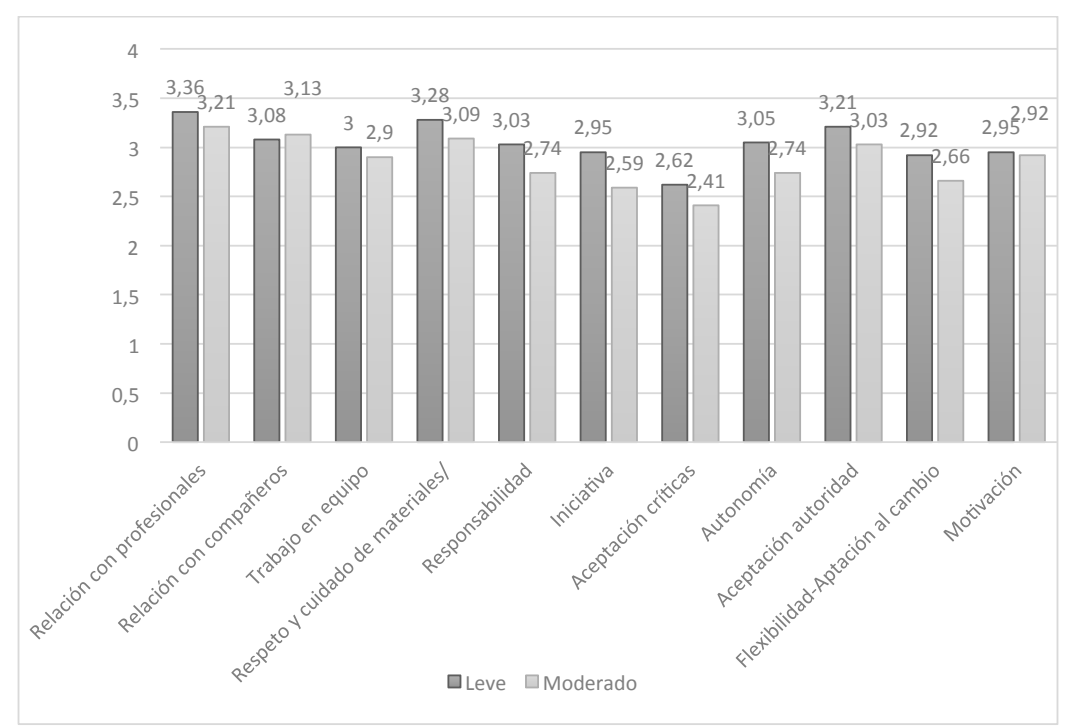

Figura 4. Competencias sociales y actitudinales.

\section{Resultados según el nivel de competencias comunicativas}

En la figura 5 se observa que todas las puntuaciones relacionadas con las competencias comunicativas están por encima de la media. Las personas con un grado de discapacidad intelectual leve tienen un nivel superior en todas las competencias. Por último, destacar que en ambos casos, los participantes muestran los niveles más elevados en las competencias en lenguaje oral, mientras que las más bajas tienen relación en ambos casos con la compresión de instrucciones escritas.

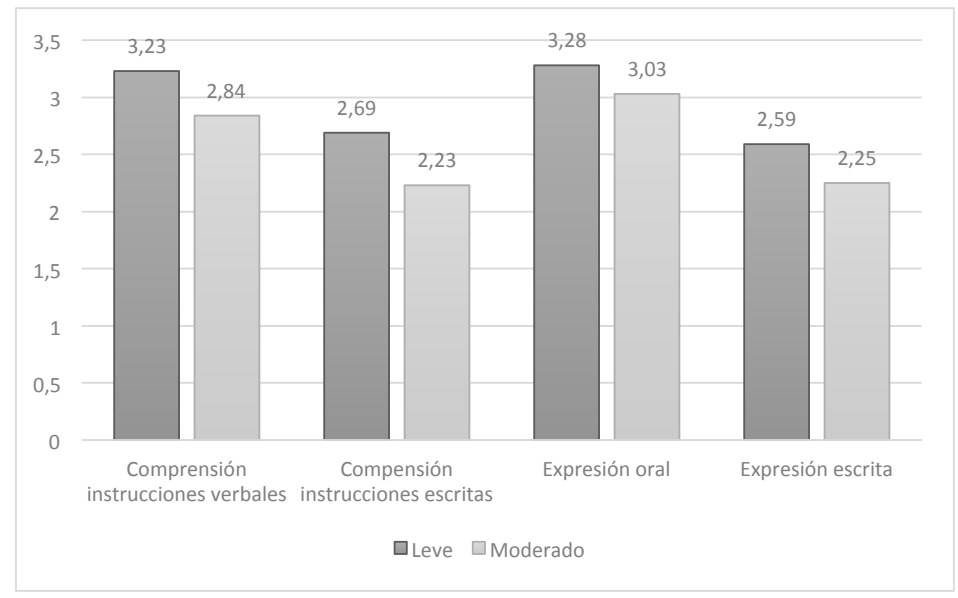

Figura 5. Competencias comunicativas. 


\section{Resultados según la relación entre niveles de desarrollo de competencias}

Los resultados que se visualizan en la tabla 3 demuestran la existencia de una relación estadísticamente significativa entre todas las competencias. En todos los casos, la relación es positiva y moderada. Ello significa que las personas que poseen niveles altos en un bloque competencial tienen igualmente niveles altos de desarrollo en las otras 3 competencias restantes.

Tabla 3

Correlación entre bloques competenciales

\begin{tabular}{|c|c|c|c|c|}
\hline \multirow[b]{2}{*}{ Competencias } & \multirow[b]{2}{*}{ Estadísticos } & \multicolumn{3}{|c|}{ Competencias } \\
\hline & & Funcionales & $\begin{array}{c}\text { Sociales y } \\
\text { actitudinales }\end{array}$ & Comunicativas \\
\hline \multirow{3}{*}{$\begin{array}{l}\text { Cognitivas y de } \\
\text { aprendizaje }\end{array}$} & $\begin{array}{l}\text { Correlación de } \\
\text { Pearson }\end{array}$ &, $667\left(^{* *}\right)$ &, $571\left(^{* *}\right)$ &, $\left.670{ }^{* *}\right)$ \\
\hline & Sig. (bilateral) & ,000 & ,000 & ,000 \\
\hline & $\mathrm{N}$ & 99 & 99 & 99 \\
\hline \multirow{3}{*}{ Funcionales } & $\begin{array}{l}\text { Correlación de } \\
\text { Pearson }\end{array}$ & &, $666\left(^{* *}\right)$ &, $541\left(^{* *}\right)$ \\
\hline & Sig. (bilateral) & & ,000 & ,000 \\
\hline & $\mathrm{N}$ & & 100 & 100 \\
\hline \multirow{3}{*}{$\begin{array}{l}\text { Sociales y } \\
\text { actitudinales }\end{array}$} & $\begin{array}{l}\text { Correlación de } \\
\text { Pearson }\end{array}$ & & &, $452\left(^{* *}\right)$ \\
\hline & Sig. (bilateral) & & & ,000 \\
\hline & $\mathrm{N}$ & & & 100 \\
\hline
\end{tabular}

\section{Resultados en función del grado de discapacidad intelectual}

Se empleó la prueba paramétrica t-test de comparación de medias para dos grupos independientes con el fin de comprobar la existencia de diferencias estadísticamente significativas según el grado de discapacidad intelectual.

Los trabajadores con un grado de DI leve demuestran un nivel general de competencias laborales superior al de los participantes con un grado de discapacidad moderado, siendo estas diferencias estadísticamente significativas $(t=2.453 ; \mathrm{g} .1 .=97 ; \mathrm{p}>.05 ; \overline{\mathrm{X}}$ grado DI leve=76.21; SD=11.66; $\overline{\mathrm{X}}$ grado DI moderado=70.60; SD=10.74). Se puede afirmar que los trabajadores con grado de DI leve muestran un nivel general de competencias laborales alto, mientras que los que tienen un grado de discapacidad moderado, demuestran niveles de competencias laborales moderadas.

Analizando independiente cada apartado del instrumento de evaluación de competencias, los participantes no muestran diferencias estadísticamente significativas en el bloque de competencias funcionales ni en el de competencias sociales y actitudinales. 
Independientemente del grado de discapacidad intelectual, el nivel alcanzado en las competencias funcionales puede considerarse alto para ambos grupos ( $\overline{\mathrm{X}}$ grado $\mathrm{DI}$ leve $=16.59$; $\mathrm{SD}=3.04 ; \overline{\mathrm{X}}$ grado $\mathrm{DI}$ moderado=15.60; $\mathrm{SD}=2.76$ ), al igual que en las competencias sociales y actitudinales $(\overline{\mathrm{X}}$ grado $\mathrm{DI}$ leve $=33.44 ; \mathrm{SD}=5.34 ; \overline{\mathrm{X}}$ grado $\mathrm{DI}$ moderado $=31.43 ; \mathrm{SD}=5.18$ ).

Donde sí se observan diferencias estadísticamente significativas es en el bloque de competencias cognitivas y de aprendizaje; así los participantes con grado de discapacidad intelectual leve demuestran niveles más altos que los de grado DI moderado ( $\mathrm{t}=2.164$; g.l.=97; $\mathrm{p}>.05 ; \overline{\mathrm{X}}$ grado DI leve=11.84; $\mathrm{SD}=2.14 ; \overline{\mathrm{X}}$ grado DI moderado=10.90; $\mathrm{SD}=2.11$ ).

También se han encontrado diferencias estadísticamente significativas en el bloque de competencias comunicativas, en este caso también son los participantes con grado de DI leve, los que demuestran mayor nivel competencial $(t=2.707 ;$ g.l. $=98 ; \mathrm{p}>.05 ; \overline{\mathrm{X}}$ grado DI leve=11.74; $\mathrm{SD}=2.73 ; \overline{\mathrm{X}}$ grado DI moderado=10.34; $\mathrm{SD}=2.38$ ).

\section{Resultados en función del género}

Los resultados competenciales genéricos en función del género que se presentan en la figura 6, muestran un mayor nivel de las mujeres en las competencias cognitivas y de aprendizaje, funcionales y comunicativas, sin tener en cuenta el grado de DI.

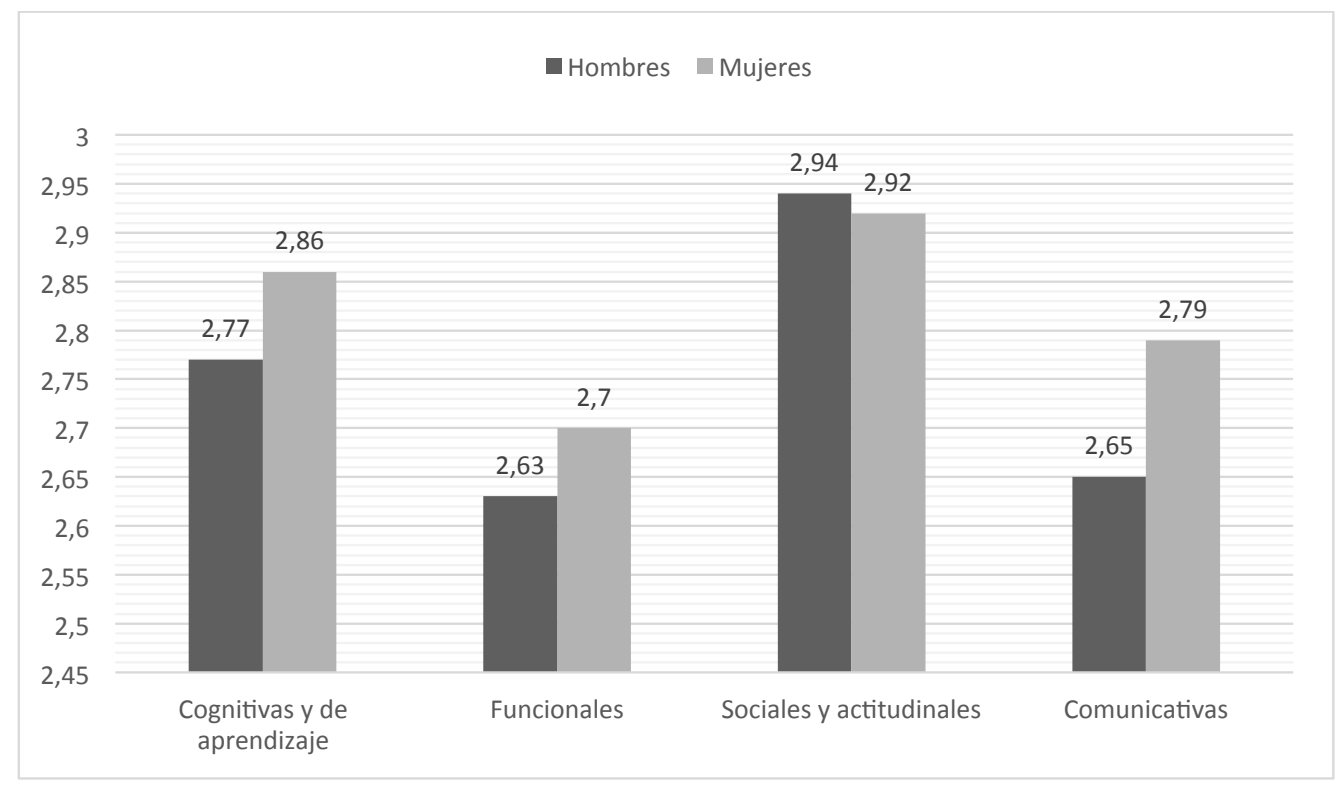

Figura 6. Niveles competenciales según la variable género.

Los resultados competenciales por bloques, tras el análisis de las puntuaciones medias obtenidas por los participantes en el estudio en función del género, muestran que las mujeres con un grado de DI leve tienen niveles competenciales superiores en todas las subescalas (figura 7). 


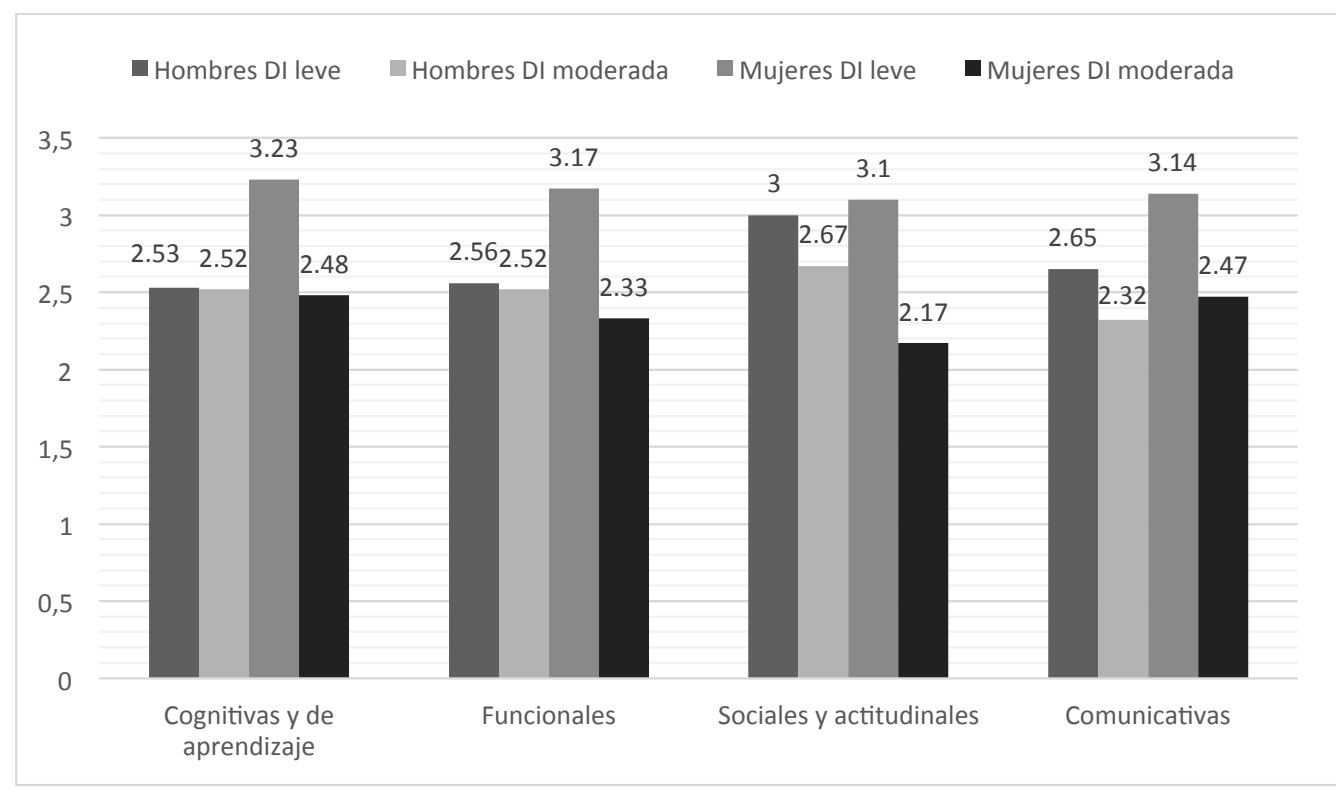

Figura 7. Nivel de competencias en función del género y el grado de DI.

Se empleó la prueba paramétrica de comparación de medias para más de dos grupos para comprobar la existencia de diferencias estadísticamente significativas entre hombres y mujeres según su grado de discapacidad. Si se analizan los datos globales (sumatorio de competencias), solo se observan diferencias estadísticamente significativas entre las mujeres. Así, las mujeres con un grado de DI leve demuestran más competencias laborales que las mujeres con un grado de DI moderado $(\mathrm{F}=2.675 ; \mathrm{p}>.05$; mujeres grado DI leve [ $\overline{\mathrm{X}}=12.36$; $\mathrm{SD}=1.97]$; mujeres grado DI moderado $[\overline{\mathrm{X}}=10.76 ; \mathrm{SD}=1.39]$ ).

$\mathrm{Al}$ analizar cada bloque de subcompetencias, los resultados apuntan la existencia de diferencias estadísticamente significativas en el apartado de competencias cognitivas y de aprendizaje entre las mujeres. De este modo, las mujeres con un grado de DI leve poseen un nivel más alto de estas competencias que las de DI moderado $(\mathrm{F}=2.794 ; \mathrm{p}>$.05; mujeres grado DI leve $[\overline{\mathrm{X}}=3.23$; $\mathrm{SD}=.92]$; mujeres grado $\mathrm{DI}$ moderado $[\overline{\mathrm{X}}=2.48 ; \mathrm{SD}=1.02]$ ).

Asimismo, se observan diferencias estadísticamente significativas entre las mujeres en el bloque de competencias sociales y actitudinales. Así, las mujeres con un grado de DI leve demuestran un nivel más alto de estas competencias que las de DI moderado $(\mathrm{F}=2,940 ; \mathrm{p}>.05$; mujeres grado DI leve $[\overline{\mathrm{X}}=3.1 ; \mathrm{SD}=1.17]$; mujeres grado DI moderado $[\overline{\mathrm{X}}=2.18 ; \mathrm{SD}=1.16])$.

También se observan diferencias estadísticamente significativas en las competencias comunicativas entre los hombres con un grado de DI moderado y las mujeres con grado de DI leve, siendo las mujeres las que demuestran mayores competencias comunicativas $(\mathrm{F}=2.854 ; \mathrm{p}>.05$; mujeres grado $\mathrm{DI}$ leve $[\overline{\mathrm{X}}=12.14 ; \mathrm{SD}=1.83]$; hombres grado DI moderado $[\overline{\mathrm{X}}=10.26 ; \mathrm{SD}=2.08])$. 


\section{Discusión y conclusiones}

La información recopilada demuestra que las personas con DI tienen un desarrollo similar en las competencias analizadas, remarcando el buen nivel obtenido en todas las competencias estudiadas para ambos grupos (usuarios con DI leve y usuarios con DI moderada). Es destacable que, independientemente del grado, todas las competencias evaluadas superen la puntuación media. Seguramente el hecho de que las personas analizadas estén ubicadas en centros ocupacionales es una variable que facilita su desarrollo competencial. Docampo y Morán (2014) coinciden en la importancia de los programas formativos desarrollados en los centros de trabajo para el éxito de estas personas, no solo en la vertiente laboral sino en todas las dimensiones de su vida.

Entrando en el análisis por bloques de competencias, se han encontrado diferencias estadísticamente significativas en dos de las cuatro dimensiones del cuestionario (las referentes a competencias cognitivas y de aprendizaje y a las comunicativas).

En cuanto a las competencias cognitivas y de aprendizaje, los resultados demuestran que las personas con DI leve poseen mayores competencias. Sin embargo, cabe resaltar que en ambos casos (DI leve y moderada) la subcompetencia más desarrollada es la de la atención selectiva. Este dato es relevante para la formación de este colectivo, puesto que, si los programas y procesos de acción pedagógica son motivadores, la atención selectiva será un elemento favorecedor para la consecución de los objetivos marcados.

Las competencias comunicativas son el segundo bloque en el que se han encontrado diferencias estadísticamente significativas. En todos los casos, las personas con DI leve son más competentes comunicativamente que las que tienen DI moderada. Sin embargo, es interesante destacar que ambos grupos muestran más desarrollada la comunicación oral, que la escrita.

El hecho de que en los ítems relativos al lenguaje oral se observen medias superiores a los relativos al lenguaje escrito, cuadra con el hecho de que en muchos casos puedan aparecer dificultades en la adquisición de la lectoescritura (Centro de Recursos de Educación Especial de Navarra, 2008).

En cuanto a la expresión escrita, también es remarcable que ambos colectivos escriban mejor que no comprendan. Es decir, que la expresión predomina ante la comprensión independientemente del medio. Autores como Puente, Alvarado, Jiménez, y Martínez (2017) apuntan las notables dificultades que tiene estas personas para desarrollar la habilidad de la comprensión lectora, y Grieco, Pulsifer, Seligsohn, Skotko, y Schwartz, (2015) consideran que dicha comprensión siempre quedará limitada. Esta dificultad objetiva en la recepción del lenguaje también es auto percibida como la mayor dificultad por parte de miembros del propio colectivo (Kumin, 2015).

Se presenta aquí otra clave relevante para la formación de personas con discapacidad intelectual. En el momento de planificar y desarrollar intervenciones y acciones formativas, el sistema prioritario de expresión debería ser el oral (dada la superioridad del medio auditivo como canal de recepción comunicativa), y siempre contando con las dificultades que tiene el colectivo para la comprensión lectora, coincidiendo con Fajardo, Tavares, Ávila y Ferrer (2013). Estos mismos autores sugieren el uso de la simplificación de textos, eliminando toda aquella información irrelevante (por ejemplo, conectores) para la facilitación del uso del soporte escrito. 
Los bloques competenciales en los que no se han encontrado diferencias significativas (el de competencias funcionales y el de competencias sociales y actitudinales), muestran tendencias que cabe exponer por su relevancia para la optimización de los aprendizajes en los centros ocupacionales.

En lo referente al análisis de las competencias funcionales, las personas con DI leve solicitan ayuda cuando la necesitan, pero muestran resistencia a la realización de tareas repetitivas, mientras que las personas con DI moderada desarrollan mejores habilidades manipulativas, pero tienen dificultades en la aplicación de estrategias para la resolución de problemas como apuntan Grieco et al. (2015).

Estos aspectos muestran disposiciones de trabajo pedagógico, tanto en lo que se refiere a puntos fuertes como a aspectos a mejorar para las personas de ambos grupos.

Para acabar el análisis independiente de los bloques competenciales, y en cuanto al análisis las competencias sociales y actitudinales, ambos grupos son coincidentes para las dimensiones más y menos desarrolladas: destacan positivamente en cuanto a la subcompetencia de relación con los profesionales de los centros ocupacionales, y tienen como dimensión menos desarrollada la de aceptación de la crítica, competencia que quizá se debería trabajar más específicamente en los centros.

Por otro lado, se evidencia la relación existente entre los cuatro tipos de competencias analizadas. Los resultados ponen de manifiesto que cada bloque incide positivamente en el desarrollo de los restantes. Este es un hecho altamente positivo en el ámbito laboral, puesto que no siempre ocurre que el uso de unas determinadas habilidades conlleve el avance progresivo de las restantes. Se puede afirmar que un puesto de trabajo que se adapte al perfil de una persona con DI será una fuente de enriquecimiento holístico de efecto positivo, puesto que propicia el desarrollo de los distintos campos competenciales (cognitivo y de aprendizaje, funcional, social y actitudinal y comunicativo).

La exploración de los datos en función del grado de discapacidad intelectual muestra que las personas que presentan un grado de DI leve manifiestan niveles competenciales superiores a los de las personas con DI moderada. Ello es lógico si se considera que el grado de discapacidad intelectual es un factor muy importante en el desarrollo integral de la persona. Estos resultados coinciden, por ejemplo, con los de estudios desarrollados en el Centro de Recursos de Educación Especial de Navarra (2008), donde se constató un mayor logro de autonomía y desarrollo en personas con DI leve. En la investigación actual solo en un caso, y no es estadísticamente significativo, el grupo con DI moderada es superior al de DI leve, y es en la subcompetencia de relación con los compañeros, dentro del bloque de competencias sociales y actitudinales. Esto contrasta con lo expuesto por De Jesús, Girón y García (2009) en torno a las mejores habilidades para entablar una conversación, menor introversión y mayor adecuación al comportamiento socialmente adaptado en personas con discapacidad intelectual leve.

En cuanto a la interrelación de los datos con la variable género, se constata un mayor nivel competencial general en las mujeres con un grado de DI leve que en las que presentan un grado de DI moderado. Los hombres por su parte muestran niveles de competencias laborales similares independientemente de su grado de DI, por lo que en éstos el grado de DI parece no incidir en el desarrollo competencial, mientras que en las mujeres sí. 
No existen diferencias en función del género en cuanto a los niveles de competencias funcionales, aunque existe una cierta tendencia entre las mujeres con grado de DI leve a manifestar un mayor nivel competencial. Cabe destacar que, entre las competencias funcionales que requieren respuestas de calidad superior, no se observan niveles inferiores comparándolas con el resto de las competencias.

$\mathrm{Al}$ analizar los 3 bloques competenciales restantes (cognitivas y de aprendizaje, sociales y actitudinales, y comunicativas), se manifiestan claras diferencias en las últimas entre hombres con DI moderado y mujeres con DI leve. Por otro lado, mientras que en los hombres parece que el grado de DI no influye en el despliegue de las competencias cognitivas y de aprendizaje y las sociales y actitudinales, entre las mujeres los niveles de DI sí lo hacen, siendo las que poseen grados de DI leves las que manifiestan mejores niveles en este tipo de competencias.

El desarrollo de las competencias cognitivas y de aprendizaje favorecen mejoras en la ejecución de las tareas, la organización del trabajo y la autonomía laboral. En esta línea los trabajos de Pallisera, Fullana y Vila (2005) y los de Becerra, Montanero, Lucero y González (2008) ponen de manifiesto la importancia de la formación en este tipo de competencias para este colectivo, con la finalidad no sólo de mejorar su capacidad de planificar, ejecutar y evaluar las tareas encomendadas, sino también para ir adquiriendo una mayor autonomía sin supervisión.

Acciones educativas decididas en cuanto a las competencias sociales y actitudinales mejorarían sin duda la autonomía de la persona en su entorno. En este sentido los trabajos desarrollados por Esteban y Jordán de Urríes (2006), muestran que es necesario potenciar habilidades sociales que puedan ayudar a la persona con DI a establecer redes sociales externas que les ayuden a integrarse plenamente en la comunidad, permitiéndoles una vida independiente en el ámbito comunitario extralaboral. De igual modo, se ha documentado la importancia que para los propios implicados tienen las redes sociales como soporte para su inclusión social (van Asselt-Goverts, Embregts y Hendri, 2015).

En cuanto a las competencias comunicativas, los programas de mejora son necesarios, sobre todo, entre los todos hombres y las mujeres con grado de DI moderado, puesto que estas competencias son imprescindibles para lograr una mayor autonomía en el entorno. Los resultados obtenidos por Pérez y Cabezas (2007), demuestran que el entrenamiento de estas competencias mejora el nivel de las sociales, necesarias igualmente en el ámbito laboral; Ello justifica la introducción de acciones para entrenar habilidades sociales específicas que mejoren este bloque competencial.

Un elemento a tener en cuenta para optimizar los procesos de inserción laboral es la necesidad de introducir actuaciones que mejoren los cuatro bloques de competencias en las mujeres con DI moderado, puesto que son las que han mostrado niveles inferiores. Asimismo, la intervención desde el ámbito laboral influiría positivamente en otros ámbitos de la vida de las personas, llegando incluso a repercutir en la calidad de vida de la familia (Foley, Girdler, Downs, Jacoby, Bourke, Lennox, Einfeld, Llewellyn, Parmenter y Leonard, 2014)

Para finalizar, cabe recordar que la plena inserción laboral en un entorno el máximo normalizado posible es un derecho fundamental de todas las personas, y que optimizando el desarrollo competencial se facilita un mayor grado de inclusión sociolaboral. Por ello 
en la presente investigación se ha destacado la importancia que las experiencias profesionalizadoras tienen para este colectivo. De igual modo, del análisis presentado también se deduce la tarea desarrollada desde los centros ocupacionales en aras a favorecer las competencias laborales de las personas con un grado de DI leve y moderado, puesto que dichos niveles competenciales se encuentran siempre por encima de la media. Asimismo, se han dado claves para optimizar los procesos formativos laborales de este colectivo, contextualizados en centros ocupacionales, en función de los resultados obtenidos. Un aumento de las competencias analizadas facilitaría el incremento del nivel de autonomía, así como de las posibilidades de una inserción más normalizada y exitosa en la vida comunitaria de las personas con DI leve y moderada.

\section{Referencias}

Ali, M., Schur, L., \& Blanck, P. (2011). What types of Jobs do people with disabilities want? Journal of Ocupational Rehabilitation, 21(2), 199-210. doi: 10.1007/s10926-010-9266-0

Alonso-Martín, P. (2010). La valoración de la importancia de las competencias transversales: comparación de su percepción al inicio y final de curso en alumnos de psicología. Revista de Investigación Educativa, 28(1), 119-140.

Arancibia Fernández, F. (2011). Flexibilidad laboral: elementos teóricos-conceptuales para su análisis. Revista de Ciencias Sociales, 26, 39-55.

Becerra, M.T., Montanero, M., Lucero, M., \& González, E. (2008). Evaluación de un programa de apoyo laboral para trabajadores con discapacidad intelectual en tareas con elevada exigencia cognitiva. Revista Española sobre Discapacidad Intelectual, 39(2), 63-81.

Castro, L., Casas, J. A., Sánchez, S., Vallejos, V., \& Zúñiga, D. (2016). Percepción de la calidad de vida en personas con discapacidad y su relación con la educación. Estudios pedagógicos 42(2), 39- 49. doi: 10.4067/S0718-07052016000200003

Centro de Recursos de Educación Especial de Navarra [CREENA] (2008). Retraso Mental. Recuerado de http://www.pnte. cfnavarra.es/creena/008psiquicos/retraso\%20mental. htm.

Comisión Europea (2008). Nuevas Capacidades para Nuevos Empleos. Previsión de las capacidades necesarias y su adecuación a las exigencias del mercado laboral. Recuperado de http://eur-lex.europa.eu/legal-content/ES/TXT/PDF/?uri=CELEX:520 08DC0868\&from=ES.

De Cuyper, N., Mauno, S., Kinnunen, U., \& Mäkikangas, A. (2011). The role of job resources in the relation between perceived employability and turnover intention: A prospective two-sample study, Journal of Vocational Behavior, 78(2), 253-263. doi: 10.1016/j.jvb.2010.09.008

De Jesús, A., Girón, I., \& García, G. (2009). Relación entre la modalidad de empleo y el nivel de calidad de vida laboral de personas con discapacidad intelectual leve. Nómadas 22(2), 333-381.

De la Fuente Anuncibay, R., \& González-Castro, J. L. (2009). Empleo, formación e inserción de colectivos en riesgo de exclusión. Un reto social y económico para la empresa receptora, Bordón, 61(3), 33-46.

Docampo Núñez, G., \& Morán de Castro, M. C. (2014). Evaluación de la competencia profesional en personas con discapacidad intelectual. Una propuesta de adaptación 
de la metodología e instrumentos INCUAL para la igualdad de oportunidades. Revista Española de Discapacidad, 2(1), 71-96. doi: 10.5569/2340-5104.02.01.04

Esteban, B. y Jordán de Urríes, F. B. (2006). Empleo con apoyo para personas con discapacidad intelectual y para personas con enfermedad mental. Comparación metodológica en dos proyectos piloto. Siglo Cero, 37(2), 63-78.

Fajardo, I., Tavares, G., Ávila, V., \& Ferrer, A. (2013) Towards text simplification for poor readers with intellectual disability: when do connectives enhance text cohesion? Research in Developmental Disabilities, 34(4), 1267-1279. doi: /10.1016/j.ridd.2013.01.006.

González, H., \& Laborda, C. (2017). Usuarios con discapacidad intelectual leve y moderada en centros ocupacionales: competencias laborales y calidad de vida. En Rodríguez-Martín, A. (Comp.). Prácticas innovadoras inclusivas: retos y oportunidades (pp. 2621-2628). Oviedo.

Flores Robaina, N, Jenaro Río, C, Garcia-Calvo, P, González-Gil, F, (2017) Análisis de la calidad de vida laboral en trabajadores con discapacidad. Revista de Servicios Sociales, 47, 95-107.

Foley, K.R., Girdler, S., Downs, J., Jacoby, P., Bourke, J., Lennox, N., Einfeld, S., Llewellyn, G., Parmenter, T. R., \& Leonard, H. (2014). Relationship between family quality of life and day occupations of young people with Down syndrome. Social Psychiatry and Psychiatric Epidemiology, 49(9), 1455-1465. doi: 10.1007/s00127-013-0812-x

Grieco, J., Pulsifer, M., Seligsohn, K., Skotko, B., \& Schwartz, A. (2015). Down syndrome: Cognitive and behavioral functioning across the lifespan. American Journal of Medical Genetics 169(2), 135-149. doi: 10.1002/ajmg.c.31439

Instituto Nacional de Empleo [INE] (2016). El empleo de las personas con discapacidad. Recuperado de http://www.ine.es/dyngs/INEbase/es/operacion.htm?c=Estadistica_C \&cid=1254736055502\&menu=ultiDatos\&idp=1254735573175.

Kumin, L. (2015). A reexamination of the receptive-expressive language gap in individuals with Down syndrome. International Medical Review on Down Syndrome, 19(2), 28-34. doi: 10.1016/j.sdeng.2015.05.002

Laborda, C. y González, H. (2017). Características diferenciales del empleo de personas con discapacidad intelectual. En Rodríguez-Martín, A. (Comp.). Prácticas innovadoras inclusivas: retos y oportunidades (pp.2647-2654). Oviedo.

Lucas Manga, S., Arias Martínez, B., \& Ovejero Bernal A. (2005). Orientación profesional e inserción sociolaboral de personas con discapacidad intelectual. Revista universitaria de ciencias del trabajo, 6, 393-414.

Martínez-Agut, M.P., \& Ramos Hernando, C. (2014). Basic Communication Skills in Professional Insertion. Intervention Grade Students of Directors and Management. Procedia - Social and Behavioral Sciences, 139, 273- 281. doi: 10.1016/j.sbspro.2014.08.074

Martínez-Clares, P., Martínez-Juárez, M. y Muñoz-Cantero, J.M. (2008). Formación basada en competencias en educación sanitaria: aproximaciones a enfoques y modelos de competencia. RELIEVE, 14 (2), 1-23. Recuperado de http:/www.uv.es/RELIEVE/ v14n2/RELIEVEv14n2_1.htm

Observatorio sobre discapacidad y mercado de trabajo en España [ODISMET] (2016). Informe 1. Resultados principales 2016. Recuperado de http://www.odismet.es/es/informes/. 
Pallisera, M., Fullana, J. \& Vila, M. (2005). La inserción laboral de personas con discapacidad. Desarrollo de tres investigaciones acerca de los factores favorecedores de los procesos de inserción. Revista de Investigación Educativa, 23(2), 295-313.

Pallisera, M., Vila, M., \& Valls, M.J. (2003). The current situation of supported employment in Spain: analysis and perspectives based on the perception of professionals. Disability \& Society, 18 (6), 797-810.

Pegalajar Palomino, M.C. \& Xandri Martínez, R. (2015). La inclusión sociolaboral de jóvenes con discapacidad intelectual: una experiencia a partir del programa ه-Capacitas®. Revista nacional e internacional de educación inclusiva, 8(1), 59-73.

Pérez Sánchez, L., \& Cabezas Gómez, D. (2007). Programa de entrenamiento en solución de problemas práctico aplicado a personas con discapacidad intelectual. Psicothema, 19(4), 578-584.

Puente, A., Alvarado, J. M., Jiménez, V., \& Martínez, L. (2017). Reading Profiles in Adolescents with Fragile X Syndrome and Down Syndrome. Anales de Psicología, 33(3), 660-669. doi: 10.6018/analesps.33.3.270721

Roselló Ramón, R., \& Verger Gelabert, S. (2008). La inclusión de las personas con discapacidad en el lugar de trabajo en las Islas Baleares. Revista Europea de Formación Profesional, 45, 181-200.

Simões, C., \& Santos, S, (2016). Comparing the quality of life of adults with and without intellectual disability. Journal of Intellectual Disability Research 60(4), 378-388. doi: 10.1111/jir.12256

van Asselt-Goverts, A. E, Embregts, P. J., \& Hendri A. (2015) Social networks of people with mild intellectual disabilities: characteristics, satisfaction, wishes and quality of life. Journal of Intellectual Disability Research, 59(5), 450-461. doi: 10.1111/jir.12143

Fecha de recepción: 27 de noviembre de 2017.

Fecha de revisión: 17 de enero de 2018.

Fecha de aceptación: 14 de octubre de 2019. 
